\title{
New Results on Multiple Solutions for Nth-Order Fuzzy Differential Equations under Generalized Differentiability
}

\author{
A. Khastan, ${ }^{1,2}$ F. Bahrami, ${ }^{1,2}$ and K. Ivaz ${ }^{1,2}$ \\ ${ }^{1}$ Department of Applied Mathematics, University of Tabriz, Tabriz 51666 16471, Iran \\ ${ }^{2}$ Research Center for Industrial Mathematics, University of Tabriz, Tabriz 51666 16471, Iran \\ Correspondence should be addressed to A. Khastan, khastan@gmail.com
}

Received 30 April 2009; Accepted 1 July 2009

Recommended by Juan José Nieto

\begin{abstract}
We firstly present a generalized concept of higher-order differentiability for fuzzy functions. Then we interpret $N$ th-order fuzzy differential equations using this concept. We introduce new definitions of solution to fuzzy differential equations. Some examples are provided for which both the new solutions and the former ones to the fuzzy initial value problems are presented and compared. We present an example of a linear second-order fuzzy differential equation with initial conditions having four different solutions.
\end{abstract}

Copyright (C) 2009 A. Khastan et al. This is an open access article distributed under the Creative Commons Attribution License, which permits unrestricted use, distribution, and reproduction in any medium, provided the original work is properly cited.

\section{Introduction}

The term "fuzzy differential equation" was coined in 1987 by Kandel and Byatt [1] and an extended version of this short note was published two years later [2]. There are many suggestions to define a fuzzy derivative and in consequence, to study fuzzy differential equation [3]. One of the earliest was to generalize the Hukuhara derivative of a set-valued function. This generalization was made by Puri and Ralescu [4] and studied by Kaleva [5]. It soon appeared that the solution of fuzzy differential equation interpreted by Hukuhara derivative has a drawback: it became fuzzier as time goes by [6]. Hence, the fuzzy solution behaves quite differently from the crisp solution. To alleviate the situation, Hüllermeier [7] interpreted fuzzy differential equation as a family of differential inclusions. The main shortcoming of using differential inclusions is that we do not have a derivative of a fuzzynumber-valued function.

The strongly generalized differentiability was introduced in [8] and studied in [911]. This concept allows us to solve the above-mentioned shortcoming. Indeed, the strongly 
generalized derivative is defined for a larger class of fuzzy-number-valued functions than the Hukuhara derivative. Hence, we use this differentiability concept in the present paper. Under this setting, we obtain some new results on existence of several solutions for $N$ thorder fuzzy differential equations. Higher-order fuzzy differential equation with Hukuhara differentiability is considered in [12] and the existence and uniqueness of solution for nonlinearities satisfying a Lipschitz condition is proved. Buckley and Feuring [13] presented two different approaches to the solvability of $N$ th-order linear fuzzy differential equations.

Here, using the concept of generalized derivative and its extension to higher-order derivatives, we show that we have several possibilities or types to define higher-order derivatives of fuzzy-number-valued functions. Then, we propose a new method to solve higher-order fuzzy differential equations based on the selection of derivative type covering all former solutions. With these ideas, the selection of derivative type in each step of derivation plays a crucial role.

\section{Preliminaries}

In this section, we give some definitions and introduce the necessary notation which will be used throughout this paper. See, for example, [6].

Definition 2.1. Let $X$ be a nonempty set. A fuzzy set $u$ in $X$ is characterized by its membership function $u: X \rightarrow[0,1]$. Thus, $u(x)$ is interpreted as the degree of membership of an element $x$ in the fuzzy set $u$ for each $x \in X$.

Let us denote by $\mathbb{R}_{F}$ the class of fuzzy subsets of the real axis (i.e., $u: \mathbb{R} \rightarrow[0,1]$ ) satisfying the following properties:

(i) $u$ is normal, that is, there exists $s_{0} \in \mathbb{R}$ such that $u\left(s_{0}\right)=1$,

(ii) $u$ is convex fuzzy set (i.e., $u(t s+(1-t) r) \geq \min \{u(s), u(r)\}$, for all $t \in[0,1], s, r \in \mathbb{R})$,

(iii) $u$ is upper semicontinuous on $\mathbb{R}$,

(iv) $c l\{s \in \mathbb{R} \mid u(s)>0\}$ is compact where $c l$ denotes the closure of a subset.

Then $\mathbb{R}_{F}$ is called the space of fuzzy numbers. Obviously, $\mathbb{R} \subset \mathbb{R}_{F}$. For $0<\alpha \leq 1$ denote $[u]^{\alpha}=\{s \in \mathbb{R} \mid u(s) \geq \alpha\}$ and $[u]^{0}=c l\{s \in \mathbb{R} \mid u(s)>0\}$. If $u$ belongs to $\mathbb{R}_{F}$, then $\alpha$-level set $[u]^{\alpha}$ is a nonempty compact interval for all $0 \leq \alpha \leq 1$. The notation

$$
[u]^{\alpha}=\left[\underline{u}^{\alpha}, \bar{u}^{\alpha}\right],
$$

denotes explicitly the $\alpha$-level set of $u$. One refers to $\underline{u}$ and $\bar{u}$ as the lower and upper branches of $u$, respectively. The following remark shows when $\left[\underline{u}^{\alpha}, \bar{u}^{\alpha}\right]$ is a valid $\alpha$-level set.

Remark 2.2 (see [6]). The sufficient conditions for $\left[\underline{u}^{\alpha}, \bar{u}^{\alpha}\right]$ to define the parametric form of a fuzzy number are as follows:

(i) $\underline{u}^{\alpha}$ is a bounded monotonic increasing (nondecreasing) left-continuous function on $(0,1]$ and right-continuous for $\alpha=0$,

(ii) $\bar{u}^{\alpha}$ is a bounded monotonic decreasing (nonincreasing) left-continuous function on $(0,1]$ and right-continuous for $\alpha=0$,

(iii) $\underline{u}^{\alpha} \leq \bar{u}^{\alpha}, 0 \leq \alpha \leq 1$. 
For $u, v \in \mathbb{R}_{F}$ and $\lambda \in \mathbb{R}$, the sum $u+v$ and the product $\lambda \cdot u$ are defined by $[u+v]^{\alpha}=$ $[u]^{\alpha}+[v]^{\alpha},[\lambda \cdot u]^{\alpha}=\lambda[u]^{\alpha}$, for all $\alpha \in[0,1]$, where $[u]^{\alpha}+[v]^{\alpha}$ means the usual addition of two intervals (subsets) of $\mathbb{R}$ and $\lambda[u]^{\alpha}$ means the usual product between a scalar and a subset of $\mathbb{R}$.

The metric structure is given by the Hausdorff distance:

$$
D: \mathbb{R}_{F} \times \mathbb{R}_{F} \longrightarrow \mathbb{R}_{+} \cup\{0\},
$$

by

$$
D(u, v)=\sup _{\alpha \in[0,1]} \max \left\{\left|\underline{u}^{\alpha}-\underline{v}^{\alpha}\right|,\left|\bar{u}^{\alpha}-\bar{v}^{\alpha}\right|\right\}
$$

The following properties are wellknown:

(i) $D(u+w, v+w)=D(u, v)$, for all $u, v, w \in \mathbb{R}_{F}$ ，

(ii) $D(k \cdot u, k \cdot v)=|k| D(u, v)$, for all $k \in \mathbb{R}, u, v \in \mathbb{R}_{F}$,

(iii) $D(u+v, w+e) \leq D(u, w)+D(v, e)$, for all $u, v, w, e \in \mathbb{R}_{F}$,

and $\left(\mathbb{R}_{F}, D\right)$ is a complete metric space.

Definition 2.3. Let $x, y \in \mathbb{R}_{F}$. If there exists $z \in \mathbb{R}_{F}$ such that $x=y+z$, then $z$ is called the $H$-difference of $x, y$ and it is denoted $x \ominus y$.

In this paper the sign " $\ominus$ " stands always for $H$-difference and let us remark that $x \ominus$ $y \neq x+(-1) y$ in general. Usually we denote $x+(-1) y$ by $x-y$, while $x \ominus y$ stands for the $H$-difference.

\section{Generalized Fuzzy Derivatives}

The concept of the fuzzy derivative was first introduced by Chang and Zadeh [14]; it was followed up by Dubois and Prade [15] who used the extension principle in their approach. Other methods have been discussed by Puri and Ralescu [4], Goetschel and Voxman [16], Kandel and Byatt [1, 2]. Lakshmikantham and Nieto introduced the concept of fuzzy differential equation in a metric space [17]. Puri and Ralescu in [4] introduced H-derivative (differentiability in the sense of Hukuhara) for fuzzy mappings and it is based on the $\mathrm{H}$ difference of sets, as follows. Henceforth, we suppose $I=\left(T_{1}, T_{2}\right)$ for $T_{1}<T_{2}, T_{1}, T_{2} \in \mathbb{R}$.

Definition 3.1. Let $F: I \rightarrow \mathbb{R}_{F}$ be a fuzzy function. One says, $F$ is differentiable at $t_{0} \in I$ if there exists an element $F^{\prime}\left(t_{0}\right) \in \mathbb{R}_{F}$ such that the limits

$$
\lim _{h \rightarrow 0^{+}} \frac{F\left(t_{0}+h\right) \ominus F\left(t_{0}\right)}{h}, \quad \lim _{h \rightarrow 0^{+}} \frac{F\left(t_{0}\right) \ominus F\left(t_{0}-h\right)}{h}
$$

exist and are equal to $F^{\prime}\left(t_{0}\right)$. Here the limits are taken in the metric space $\left(\mathbb{R}_{F}, D\right)$. 
The above definition is a straightforward generalization of the Hukuhara differentiability of a set-valued function. From [6, Proposition 4.2.8], it follows that Hukuhara differentiable function has increasing length of support. Note that this definition of derivative is very restrictive; for instance, in [9], the authors showed that if $F(t)=c \cdot g(t)$, where $c$ is a fuzzy number and $g:[a, b] \rightarrow \mathbb{R}^{+}$is a function with $g^{\prime}(t)<0$, then $F$ is not differentiable. To avoid this difficulty, the authors [9] introduced a more general definition of derivative for fuzzy-number-valued function. In this paper, we consider the following definition [11].

Definition 3.2. Let $F: I \rightarrow \mathbb{R}_{F}$ and fix $t_{0} \in I$. One says $F$ is (1)-differentiable at $t_{0}$, if there exists an element $F^{\prime}\left(t_{0}\right) \in \mathbb{R}_{F}$ such that for all $h>0$ sufficiently near to 0 , there exist $F\left(t_{0}+\right.$ $h) \ominus F\left(t_{0}\right), F\left(t_{0}\right) \ominus F\left(t_{0}-h\right)$, and the limits (in the metric $D$ )

$$
\lim _{h \rightarrow 0^{+}} \frac{F\left(t_{0}+h\right) \ominus F\left(t_{0}\right)}{h}=\lim _{h \rightarrow 0^{+}} \frac{F\left(t_{0}\right) \ominus F\left(t_{0}-h\right)}{h}=F^{\prime}\left(t_{0}\right) .
$$

$F$ is (2)-differentiable if for all $h<0$ sufficiently near to 0 , there exist $F\left(t_{0}+h\right) \ominus F\left(t_{0}\right), F\left(t_{0}\right) \ominus$ $F\left(t_{0}-h\right)$ and the limits (in the metric $D$ )

$$
\lim _{h \rightarrow 0^{-}} \frac{F\left(t_{0}+h\right) \ominus F\left(t_{0}\right)}{h}=\lim _{h \rightarrow 0^{-}} \frac{F\left(t_{0}\right) \ominus F\left(t_{0}-h\right)}{h}=F^{\prime}\left(t_{0}\right)
$$

If $F$ is $(n)$-differentiable at $t_{0}$, we denote its first derivatives by $D_{n}^{(1)} F\left(t_{0}\right)$, for $n=1,2$.

Example 3.3. Let $g: I \rightarrow \mathbb{R}^{+}$and define $f: I \rightarrow \mathbb{R}_{F}$ by $f(t)=c \cdot g(t)$, for all $t \in I$. If $g$ is differentiable at $t_{0} \in I$, then $f$ is generalized differentiable on $t_{0} \in I$ and we have $f^{\prime}\left(t_{0}\right)=c$. $g^{\prime}\left(t_{0}\right)$. For instance, if $g^{\prime}\left(t_{0}\right)>0, f$ is (1)-differentiable. If $g^{\prime}\left(t_{0}\right)<0$, then $f$ is (2)-differentiable.

Remark 3.4. In the previous definition, (1)-differentiability corresponds to the H-derivative introduced in [4], so this differentiability concept is a generalization of the H-derivative and obviously more general. For instance, in the previous example, for $f(t)=c \cdot g(t)$ with $g^{\prime}\left(t_{0}\right)<$ 0 , we have $f^{\prime}\left(t_{0}\right)=c \cdot g^{\prime}\left(t_{0}\right)$.

Remark 3.5. In [9], the authors consider four cases for derivatives. Here we only consider the two first cases of [9, Definition 5]. In the other cases, the derivative is trivial because it is reduced to crisp element (more precisely, $F^{\prime}\left(t_{0}\right) \in \mathbb{R}$. For details, see [9, Theorem 7]).

Theorem 3.6. Let $F: I \rightarrow \mathbb{R}_{F}$ be fuzzy function, where $[F(t)]^{\alpha}=\left[f_{\alpha}(t), g_{\alpha}(t)\right]$ for each $\alpha \in[0,1]$.

(i) If $F$ is (1)-differentiable, then $f_{\alpha}$ and $g_{\alpha}$ are differentiable functions and $\left[D_{1}^{1} F(t)\right]^{\alpha}=$ $\left[f_{\alpha}^{\prime}(t), g_{\alpha}^{\prime}(t)\right]$.

(ii) If $F$ is (2)-differentiable, then $f_{\alpha}$ and $g_{\alpha}$ are differentiable functions and $\left[D_{2}^{1} F(t)\right]^{\alpha}=$ $\left.\left[g_{\alpha}^{\prime}(t), f_{\alpha}^{\prime}(t)\right)\right]$

Proof. See [11].

Now we introduce definitions for higher-order derivatives based on the selection of derivative type in each step of differentiation. For the sake of convenience, we concentrate on the second-order case. 
For a given fuzzy function $F$, we have two possibilities (Definition 3.2) to obtain the derivative of $F$ ot $t: D_{1}^{(1)} F(t)$ and $D_{2}^{(1)} F(t)$. Then for each of these two derivatives, we have again two possibilities: $D_{1}^{(1)}\left(D_{1}^{(1)} F(t)\right), D_{2}^{(1)}\left(D_{1}^{(1)} F(t)\right)$, and $D_{1}^{(1)}\left(D_{2}^{(1)} F(t)\right), D_{2}^{(1)}\left(D_{2}^{(1)} F(t)\right)$, respectively.

Definition 3.7. Let $F: I \rightarrow \mathbb{R}_{F}$ and $n, m=1,2$. One says say $F$ is $(n, m)$-differentiable at $t_{0} \in I$, if $D_{n}^{(1)} F$ exists on a neighborhood of $t_{0}$ as a fuzzy function and it is $(m)$-differentiable at $t_{0}$. The second derivatives of $F$ are denoted by $D_{n, m}^{(2)} F\left(t_{0}\right)$ for $n, m=1,2$.

Remark 3.8. This definition is consistent. For example, if $F$ is $(1,2)$ and $(2,1)$-differentiable simultaneously at $t_{0}$, then $F$ is (1)- and (2)-differentiable around $t_{0}$. By remark in [9], $F$ is a crisp function in a neighborhood of $t_{0}$.

Theorem 3.9. Let $D_{1}^{(1)} F: I \rightarrow \mathbb{R}_{F}$ or $D_{2}^{(1)} F: I \rightarrow \mathbb{R}_{F}$ be fuzzy functions, where $[F(t)]^{\alpha}=$ $\left[f_{\alpha}(t), g_{\alpha}(t)\right]$.

(i) If $D_{1}^{(1)} F$ is (1)-differentiable, then $f_{\alpha}^{\prime}$ and $g_{\alpha}^{\prime}$ are differentiable functions and $\left[D_{1,1}^{(2)} F(t)\right]^{\alpha}=$ $\left[f_{\alpha}^{\prime \prime}(t), g_{\alpha}^{\prime \prime}(t)\right]$.

(ii) If $D_{1}^{(1)} F$ is (2)-differentiable, then $f_{\alpha}^{\prime}$ and $g_{\alpha}^{\prime}$ are differentiable functions and $\left[D_{1,2}^{(2)} F(t)\right]^{\alpha}=$ $\left[g_{\alpha}^{\prime \prime}(t), f_{\alpha}^{\prime \prime}(t)\right]$.

(iii) If $D_{2}^{(1)} F$ is (1)-differentiable, then $f_{\alpha}^{\prime}$ and $g_{\alpha}^{\prime}$ are differentiable functions and $\left[D_{2,1}^{(2)} F(t)\right]^{\alpha}=$ $\left[g_{\alpha}^{\prime \prime}(t), f_{\alpha}^{\prime \prime}(t)\right]$.

(iv) If $D_{2}^{(1)} F$ is (2)-differentiable, then $f_{\alpha}^{\prime}$ and $g_{\alpha}^{\prime}$ are differentiable functions and $\left[D_{2,2}^{(2)} F(t)\right]^{\alpha}=$ $\left[f_{\alpha}^{\prime \prime}(t), g_{\alpha}^{\prime \prime}(t)\right]$.

Proof. We present the details only for the case (i), since the other cases are analogous.

If $h>0$ and $\alpha \in[0,1]$, we have

$$
\left[D_{1}^{(1)} F(t+h) \ominus D_{1}^{(1)} F(t)\right]^{\alpha}=\left[f_{\alpha}^{\prime}(t+h)-f_{\alpha}^{\prime}(t), g_{\alpha}^{\prime}(t+h)-g_{\alpha}^{\prime}(t)\right],
$$

and multiplying by $1 / h$, we have

$$
\frac{\left[D_{1}^{(1)} F(t+h) \ominus D_{1}^{(1)} F(t)\right]^{\alpha}}{h}=\left[\frac{f_{\alpha}^{\prime}(t+h)-f_{\alpha}^{\prime}(t)}{h}, \frac{g_{\alpha}^{\prime}(t+h)-g_{\alpha}^{\prime}(t)}{h}\right] .
$$

Similarly, we obtain

$$
\frac{\left[D_{1}^{(1)} F(t) \ominus D_{1}^{(1)} F(t-h)\right]^{\alpha}}{h}=\left[\frac{f_{\alpha}^{\prime}(t)-f_{\alpha}^{\prime}(t-h)}{h}, \frac{g_{\alpha}^{\prime}(t)-g_{\alpha}^{\prime}(t-h)}{h}\right] .
$$


Passing to the limit, we have

$$
\left[D_{1,1}^{(2)} F(t)\right]^{\alpha}=\left[f_{\alpha}^{\prime \prime}(t), g_{\alpha}^{\prime \prime}(t)\right]
$$

This completes the proof of the theorem.

Let $N$ be a positive integer number, pursuing the above-cited idea, we write $D_{k_{1}, \ldots, k_{N}}^{(N)} F\left(t_{0}\right)$ to denote the $N$ th-derivatives of $F$ at $t_{0}$ with $k_{i}=1,2$ for $i=1, \ldots, N$. Now we intend to compute the higher derivatives (in generalized differentiability sense) of the $H$-difference of two fuzzy functions and the product of a crisp and a fuzzy function.

Lemma 3.10. If $f, g: I \rightarrow \mathbb{R}_{F}$ are $N$ th-order generalized differentiable at $t \in I$ in the same case of differentiability, then $f+g$ is generalized differentiable of order $N$ at $t$ and $(f+g)^{(N)}(t)=f^{(N)}(t)+$ $g^{(N)}(t)$. (The sum of two functions is defined pointwise.)

Proof. By Definition 3.2 the statement of the lemma follows easily.

Theorem 3.11. Let $f, g: I \rightarrow \mathbb{R}_{F}$ be second-order generalized differentiable such that $f$ is $(1,1)$ differentiable and $g$ is $(2,1)$-differentiable or $f$ is (1,2)-differentiable and $g$ is $(2,2)$-differentiable or $f$ is $(2,1)$-differentiable and $g$ is $(1,1)$-differentiable or $f$ is $(2,2)$-differentiable and $g$ is $(1,2)$-differentiable on I. If the $H$-difference $f(t) \ominus g(t)$ exists for $t \in I$, then $f \ominus g$ is second-order generalized differentiable and

$$
(f \ominus g)^{\prime \prime}(t)=f^{\prime \prime}(t)+(-1) \cdot g^{\prime \prime}(t)
$$

for all $t \in I$.

Proof. We prove the first case and other cases are similar. Since $f$ is (1)-differentiable and $g$ is (2)-differentiable on $I$, by [10, Theorem 4], $(f \ominus g)(t)$ is (1)-differentiable and we have $(f \ominus g)^{\prime}(t)=f^{\prime}(t)+(-1) \cdot g^{\prime}(t)$. By differentiation as (1)-differentiability in Definition 3.2 and using Lemma 3.10, we get $(f \ominus g)(t)$ is $(1,1)$-differentiable and we deduce

$$
(f \ominus g)^{\prime \prime}(t)=\left(f^{\prime}(t)+(-1) \cdot g^{\prime}(t)\right)^{\prime}=f^{\prime \prime}(t)+(-1) \cdot g^{\prime \prime}(t) .
$$

The $H$-difference of two functions is understood pointwise.

Theorem 3.12. Let $f: I \rightarrow \mathbb{R}$ and $g: I \rightarrow \mathbb{R}_{F}$ be two differentiable functions ( $g$ is generalized differentiable as in Definition 3.2).

(i) If $f(t) \cdot f^{\prime}(t)>0$ and $g$ is (1)-differentiable, then $f \cdot g$ is (1)-differentiable and

$$
(f \cdot g)^{\prime}(t)=f^{\prime}(t) \cdot g(t)+f(t) \cdot g^{\prime}(t) .
$$


(ii) If $f(t) \cdot f^{\prime}(t)<0$ and $g$ is (2)-differentiable, then $f \cdot g$ is (2)-differentiable and

$$
(f \cdot g)^{\prime}(t)=f^{\prime}(t) \cdot g(t)+f(t) \cdot g^{\prime}(t)
$$

Proof. See [10].

Theorem 3.13. Let $f: I \rightarrow \mathbb{R}$ and $g: I \rightarrow \mathbb{R}_{F}$ be second-order differentiable functions ( $g$ is generalized differentiable as in Definition 3.7).

(i) If $f(t) \cdot f^{\prime}(t)>0, f^{\prime}(t) \cdot f^{\prime \prime}(t)>0$, and $g$ is $(1,1)$-differentiable then $f \cdot g$ is $(1,1)$-differentiable and

$$
(f \cdot g)^{\prime \prime}(t)=f^{\prime \prime}(t) \cdot g(t)+2 f^{\prime}(t) \cdot g^{\prime}(t)+f(t) \cdot g^{\prime \prime}(t)
$$

(ii) If $f(t) \cdot f^{\prime}(t)<0, f^{\prime}(t) \cdot f^{\prime \prime}(t)<0$ and $g$ is $(2,2)$-differentiable then $f \cdot g$ is $(2,2)$-differentiable and

$$
(f \cdot g)^{\prime \prime}(t)=f^{\prime \prime}(t) \cdot g(t)+2 f^{\prime}(t) \cdot g^{\prime}(t)+f(t) \cdot g^{\prime \prime}(t)
$$

Proof. We prove (i), and the proof of another case is similar. If $f(t) \cdot f^{\prime}(t)>0$ and $g$ is (1)differentiable, then by Theorem 3.12 we have

$$
(f \cdot g)^{\prime}(t)=f^{\prime}(t) \cdot g(t)+f(t) \cdot g^{\prime}(t)
$$

Now by differentiation as first case in Definition 3.2, since $g^{\prime}(t)$ is (1)-differentiable and $f^{\prime}(t)$. $f^{\prime \prime}(t)>0$, then we conclude the result.

Remark 3.14. By [9, Remark 16], let $f: I \rightarrow \mathbb{R}, \gamma \in \mathbb{R}_{F}$ and define $F: I \rightarrow \mathbb{R}_{F}$ by $F(t)=\gamma \cdot f(t)$, for all $t \in I$. If $f$ is differentiable on $I$, then $F$ is differentiable on $I$, with $F^{\prime}(t)=\gamma \cdot f^{\prime}(t)$. By Theorem 3.12, if $f(t) \cdot f^{\prime}(t)>0$, then $F$ is (1)-differentiable on $I$. Also if $f(t) \cdot f^{\prime}(t)<0$, then $F$ is (2)-differentiable on $I$. If $f(t) \cdot f^{\prime}(t)=0$, by [9, Theorem 10], we have $F^{\prime}(t)=\gamma \cdot f^{\prime}(t)$. We can extend this result to second-order differentiability as follows.

Theorem 3.15. Let $f: I \rightarrow \mathbb{R}$ be twice differentiable on $I, \gamma \in \mathbb{R}_{F}$ and define $F: I \rightarrow \mathbb{R}_{F}$ by $F(t)=\gamma \cdot f(t)$, for all $t \in I$.

(i) If $f(t) \cdot f^{\prime}(t)>0$ and $f^{\prime}(t) \cdot f^{\prime \prime}(t)>0$, then $F(t)$ is (1,1)-differentiable and its second derivative, $D_{1,1}^{(2)} F$, is $F^{\prime \prime}(t)=\gamma \cdot f^{\prime \prime}(t)$,

(ii) If $f(t) \cdot f^{\prime}(t)>0$ and $f^{\prime}(t) \cdot f^{\prime \prime}(t)<0$, then $F(t)$ is (1,2)-differentiable with $\left.D_{1,2}^{(2)} F\right)=$ $\gamma \cdot f^{\prime \prime}(t)$

(iii) If $f(t) \cdot f^{\prime}(t)<0$ and $f^{\prime}(t) \cdot f^{\prime \prime}(t)>0$, then $F(t)$ is $(2,1)$-differentiable with $D_{2,1}^{(2)} F=\gamma \cdot f^{\prime \prime}(t)$,

(iv) If $f(t) \cdot f^{\prime}(t)<0$ and $f^{\prime}(t) \cdot f^{\prime \prime}(t)<0$, then $F(t)$ is $(2,2)$-differentiable with $D_{2,2}^{(2)} F=r \cdot f^{\prime \prime}(t)$. 
Proof. Cases (i) and (iv) follow from Theorem 3.13. To prove (ii), since $f(t) \cdot f^{\prime}(t)>0$, by Remark 3.14, $F$ is (1)-differentiable and we have $D_{1}^{(1)} F=\gamma \cdot f^{\prime}(t)$ on $I$. Also, since $f^{\prime}(t) \cdot f^{\prime \prime}(t)<$ 0 , then $D_{1}^{(1)} F$ is (2)-differentiable and we conclude the result. Case (iii) is similar to previous one.

Example 3.16. If $\gamma$ is a fuzzy number and $\phi:(0,3) \rightarrow R$, where

$$
\phi(t)=t^{2}-3 t+2
$$

is crisp second-order polynomial, then for

$$
F(t)=\gamma \cdot \phi(t)
$$

we have the following

(i) for $0<t<1$ : $\phi(t) \cdot \phi^{\prime}(t)<0$ and $\phi^{\prime}(t) \cdot \phi^{\prime \prime}(t)<0$ then by (iv), $F(t)$ is (2-2)differentiable and its second derivative, $D_{2,2}^{(2)} F$ is $F^{\prime \prime}(t)=2 \cdot \gamma$,

(ii) for $1<t<3 / 2$ : $\phi(t) \cdot \phi^{\prime}(t)>0$ and $\phi^{\prime}(t) \cdot \phi^{\prime \prime}(t)<0$ then by (ii), $F(t)$ is (1-2)differentiable with $D_{1,2}^{(2)} F=2 \cdot \gamma$,

(iii) for 3/2<t<2: $\phi(t) \cdot \phi^{\prime}(t)<0$ and $\phi^{\prime}(t) \cdot \phi^{\prime \prime}(t)>0$ then by (iii), $F(t)$ is (2-1)differentiable and $D_{2,1}^{(2)} F=2 \cdot \gamma$,

(iv) for $2<t<3$ : $\phi(t) \cdot \phi^{\prime}(t)>0$ and $\phi^{\prime}(t) \cdot \phi^{\prime \prime}(t)>0$ then by (i), $F(t)$ is (1-1)-differentiable and $D_{1,1}^{(2)} F=2 \cdot \gamma$,

(v) for $t=1,3 / 2$, 2: we have $\phi^{\prime}(t) \cdot \phi^{\prime \prime}(t)=0$, then by [9, Theorem 10] we have $F^{\prime}(t)=$ $\gamma \cdot \phi^{\prime}(t)$, again by applying this theorem, we get $F^{\prime \prime}(t)=2 \cdot \gamma$.

\section{Second-Order Fuzzy Differential Equations}

In this section, we study the fuzzy initial value problem for a second-order linear fuzzy differential equation:

$$
\left\{\begin{array}{l}
y^{\prime \prime}(t)+a \cdot y^{\prime}(t)+b \cdot y(t)=\sigma(t) \\
y(0)=\gamma_{0} \\
y^{\prime}(0)=\gamma_{1}
\end{array}\right.
$$

where $a, b>0, \gamma_{0}, \gamma_{1} \in \mathbb{R}_{F}$, and $\sigma(t)$ is a continuous fuzzy function on some interval $I$. The interval $I$ can be $(0, A)$ for some $A>0$ or $I=(0, \infty)$. In this paper, we suppose $a, b>0$. Our strategy of solving (4.1) is based on the selection of derivative type in the fuzzy differential equation. We first give the following definition for the solutions of (4.1).

Definition 4.1. Let $y: I \rightarrow \mathbb{R}_{F}$ be a fuzzy function and $n, m \in\{1,2\}$. One says $y$ is an $(n, m)$ solution for problem (4.1) on $I$, if $D_{n}^{1} y D_{n, m}^{2} y$ exist on $I$ and $D_{n, m}^{2} y(t)+a \cdot D_{n}^{1} y(t)+b \cdot y(t)=$ $\sigma(t), y(0)=\gamma_{0}, D_{n}^{1} y(0)=\gamma_{1}$. 
Let $y$ be an $(n, m)$-solution for (4.1). To find it, utilizing Theorems 3.6 and 3.9 and considering the initial values, we can translate problem (4.1) to a system of secondorder linear ordinary differential equations hereafter, called corresponding $(n, m)$-system for problem (4.1).

Therefore, four ODEs systems are possible for problem (4.1), as follows:

$(1,1)$-system

$$
\left\{\begin{array}{l}
\underline{y}^{\prime \prime}(t ; \alpha)+a \underline{y}^{\prime}(t ; \alpha)+b \underline{y}(t ; \alpha)=\underline{\sigma}(t ; \alpha), \\
\bar{y}^{\prime \prime}(t ; \alpha)+a \bar{y}^{\prime}(t ; \alpha)+b \bar{y}(t ; \alpha)=\bar{\sigma}(t ; \alpha), \\
\underline{y}(0 ; \alpha)=\underline{\gamma}^{\alpha}, \bar{y}(0 ; \alpha)=\bar{\gamma}_{0}{ }^{\alpha}, \\
\underline{y}^{\prime}(0 ; \alpha)={\underline{\gamma_{1}}}^{\alpha}, \bar{y}^{\prime}(0 ; \alpha)={\overline{\gamma_{1}}}^{\alpha},
\end{array}\right.
$$

$(1,2)$-system

$$
\left\{\begin{array}{l}
\bar{y}^{\prime \prime}(t ; \alpha)+a \underline{y}^{\prime}(t ; \alpha)+b \underline{y}(t ; \alpha)=\underline{\sigma}(t ; \alpha), \\
\underline{y}^{\prime \prime}(t ; \alpha)+a \bar{y}^{\prime}(t ; \alpha)+b \bar{y}(t ; \alpha)=\bar{\sigma}(t ; \alpha), \\
\underline{y}(0 ; \alpha)=\underline{\gamma}^{\alpha}, \bar{y}(0 ; \alpha)=\bar{\gamma}_{0}^{\alpha}, \\
\underline{y}^{\prime}(0 ; \alpha)={\underline{\gamma_{1}}}^{\alpha}, \bar{y}^{\prime}(0 ; \alpha)={\overline{\gamma_{1}}}^{\alpha},
\end{array}\right.
$$

$(2,1)$-system

$$
\left\{\begin{array}{l}
\bar{y}^{\prime \prime}(t ; \alpha)+a \bar{y}^{\prime}(t ; \alpha)+b \underline{y}(t ; \alpha)=\underline{\sigma}(t ; \alpha), \\
\underline{y}^{\prime \prime}(t ; \alpha)+a \underline{y}^{\prime}(t ; \alpha)+b \bar{y}(t ; \alpha)=\bar{\sigma}(t ; \alpha), \\
\underline{y}(0 ; \alpha)=\underline{\gamma}^{\alpha}, \bar{y}(0 ; \alpha)=\bar{\gamma}_{0}^{\alpha}, \\
\bar{y}(0 ; \alpha)=\underline{\gamma_{1}}, \underline{y}^{\prime}(0 ; \alpha)=\bar{\gamma}_{1}
\end{array}\right.
$$

$(2,2)$-system

$$
\left\{\begin{array}{l}
\underline{y}^{\prime \prime}(t ; \alpha)+a \bar{y}^{\prime}(t ; \alpha)+b \underline{y}(t ; \alpha)=\underline{\sigma}(t ; \alpha), \\
\bar{y}^{\prime \prime}(t ; \alpha)+a \underline{y}^{\prime}(t ; \alpha)+b \bar{y}(t ; \alpha)=\bar{\sigma}(t ; \alpha), \\
\underline{y}(0 ; \alpha)={\underline{\gamma_{0}}}^{\alpha}, \bar{y}(0 ; \alpha)={\overline{\gamma_{0}}}^{\alpha}, \\
\bar{y}^{\prime}(0 ; \alpha)={\underline{\gamma_{1}}}^{\alpha}, \underline{y}^{\prime}(0 ; \alpha)={\overline{\gamma_{1}}}^{\alpha} .
\end{array}\right.
$$


Theorem 4.2. Let $n, m \in\{1,2\}$ and $y=[\underline{y}, \bar{y}]$ be an $(n, m)$-solution for problem (4.1) on I. Then $\underline{y}$ and $\bar{y}$ solve the associated $(n, m)$-systems.

Proof. Suppose $y$ is the $(n, m)$-solution of problem (4.1). According to the Definition 4.1, then $D_{n}^{1} y$ and $D_{n, m}^{2} y$ exist and satisfy problem (4.1). By Theorems 3.6 and 3.9 and substituting $y, \bar{y}$ and their derivatives in problem $(4.1)$, we get the $(n, m)$-system corresponding to $(n, m)$ solution. This completes the proof.

Theorem 4.3. Let $n, m \in\{1,2\}$ and $f_{\alpha}(t)$ and $g_{\alpha}(t)$ solve the $(n, m)$-system on $I$, for every $\alpha \in$ $[0,1]$. Let $[F(t)]^{\alpha}=\left[f_{\alpha}(t), g_{\alpha}(t)\right]$. If $F$ has valid level sets on $I$ and $D_{n, m}^{2} F$ exists, then $F$ is an $(n, m)$-solution for the fuzzy initial value problem (4.1).

Proof. Since $[F(t)]^{\alpha}=\left[f_{\alpha}(t), g_{\alpha}(t)\right]$ is $(n, m)$-differentiable fuzzy function, by Theorems 3.6 and 3.9 we can compute $D_{n}^{1} F$ and $D_{n, m}^{2} F$ according to $f_{\alpha}^{\prime}, g_{\alpha}^{\prime}, f_{\alpha}^{\prime \prime}, g_{\alpha}^{\prime \prime}$. Due to the fact that $f_{\alpha}, g_{\alpha}$ solve $(n, m)$-system, from Definition 4.1 , it comes that $F$ is an $(n, m)$-solution for (4.1).

The previous theorems illustrate the method to solve problem (4.1). We first choose the type of solution and translate problem (4.1) to a system of ordinary differential equations. Then, we solve the obtained ordinary differential equations system. Finally we find such a domain in which the solution and its derivatives have valid level sets and using Stacking Theorem [5] we can construct the solution of the fuzzy initial value problem (4.1).

Remark 4.4. We see that the solution of fuzzy differential equation (4.1) depends upon the selection of derivatives. It is clear that in this new procedure, the unicity of the solution is lost, an expected situation in the fuzzy context. Nonetheless, we can consider the existence of four solutions as shown in the following examples.

Example 4.5. Let us consider the following second-order fuzzy initial value problem

$$
y^{\prime \prime}(t)=\sigma_{0}, \quad y(0)=\gamma_{0}, \quad y^{\prime}(0)=\gamma_{1}, \quad t \geq 0,
$$

where $\sigma_{0}=\gamma_{0}=\gamma_{1}$ are the triangular fuzzy number having $\alpha$-level sets $[\alpha-1,1-\alpha]$.

If $y$ is $(1,1)$-solution for the problem, then

$$
\left[y^{\prime}(t)\right]^{\alpha}=\left[\underline{y}^{\prime}(t ; \alpha), \bar{y}^{\prime}(t ; \alpha)\right], \quad\left[y^{\prime \prime}(t)\right]^{\alpha}=\left[\underline{y}^{\prime \prime}(t ; \alpha), \bar{y}^{\prime \prime}(t ; \alpha)\right]
$$

and they satisfy $(1,1)$-system associated with (4.1). On the other hand, by ordinary differential theory, the corresponding $(1,1)$-system has only the following solution:

$$
\underline{y}(t ; \alpha)=(\alpha-1)\left(\frac{t^{2}}{2}+t+1\right), \quad \bar{y}(t ; \alpha)=(1-\alpha)\left(\frac{t^{2}}{2}+t+1\right) .
$$

We see that $[y(t)]^{\alpha}=[\underline{y}(t ; \alpha), \bar{y}(t ; \alpha)]$ are valid level sets for $t \geq 0$ and

$$
y=[\alpha-1,1-\alpha] \cdot\left(\frac{t^{2}}{2}+t+1\right)
$$


By Theorem 3.15, $y$ is $(1,1)$-differentiable for $t \geq 0$. Therefore, $y$ defines a $(1,1)$-solution for $t \geq 0$.

For $(1,2)$-solution, we get the following solutions for $(1,2)$-system:

$$
\underline{y}(t ; \alpha)=(\alpha-1)\left(-\frac{t^{2}}{2}+t+1\right), \quad \bar{y}(t ; \alpha)=(1-\alpha)\left(-\frac{t^{2}}{2}+t+1\right),
$$

where $y(t)$ has valid level sets for $t \in[0,1]$. How ever-also $[y(t)]^{\alpha}=[\alpha-1,1-\alpha] \cdot\left(-\left(t^{2} / 2\right)+t+1\right)$ where $y$ is $(1,2)$-differentiable. Then $y$ gives us a $(1,2)$-solution on $(0,1)$.

$(2,1)$-system yields

$$
\underline{y}(t ; \alpha)=(\alpha-1)\left(-\frac{t^{2}}{2}-t+1\right), \quad \bar{y}(t ; \alpha)=(1-\alpha)\left(-\frac{t^{2}}{2}-t+1\right),
$$

where $y(t)$ has valid level sets for $t \in[0, \sqrt{3}-1]$. We can see $y$ is a $(2,1)$-solution on $(0, \sqrt{3}-1)$

Finally, (2-2)-system gives

$$
\underline{y}(t ; \alpha)=(\alpha-1)\left(\frac{t^{2}}{2}-t+1\right), \quad \bar{y}(t ; \alpha)=(1-\alpha)\left(\frac{t^{2}}{2}-t+1\right)
$$

where $y(t)$ has valid level sets for all $t \in[0,1]$, and defines a $(2,2)$-solution on $(0,1)$.

Then we have an example of a second-order fuzzy initial value problem with four different solutions.

Example 4.6. Consider the fuzzy initial value problem:

$$
y^{\prime \prime}(t)+y(t)=\sigma_{0}, \quad y(0)=\gamma_{0}, \quad y^{\prime}(0)=\gamma_{1} \quad \forall t \geq 0,
$$

where $\sigma_{0}$ is the fuzzy number having $\alpha$-level sets $=[\alpha, 2-\alpha]$ and $\left[\gamma_{0}\right]^{\alpha}=\left[\gamma_{1}\right]^{\alpha}=[\alpha-1,1-\alpha]$.

To find (1,1)-solution, we have

$$
\underline{y}(t ; \alpha)=\alpha(1+\sin t)-\sin t-\cos t, \quad \bar{y}(t ; \alpha)=(2-\alpha)(1+\sin t)-\sin t-\cos t,
$$

where $y(t)$ has valid level sets for $t \geq 0$ and $y(t)=\sigma_{0} \cdot(1+\sin t)-\sin t-\cos t$. From Theorem 3.15, $y$ is $(1,2)$-differentiable on $(0, \pi / 2)$, then by Remark $3.8, y$ is not $(1,1)$-differentiable on $(0, \pi / 2)$. Hence, no $(1,1)$-solution exists for $t>0$.

For $(1,2)$-solutions we deduce

$$
\begin{aligned}
& \underline{y}(t ; \alpha)=\alpha(1+\sinh t)-\sinh t-\cos t, \\
& \bar{y}(t ; \alpha)=(2-\alpha)(1+\sinh t)-\sinh t-\cos t,
\end{aligned}
$$

we see that $y(t)$ has valid level sets and is $(1,1)$-differentiable for $t>0$. Since the $(1,2)$-system has only the above solution, then (1,2)-solution does not exist. 
For $(2,1)$-solutions we get

$$
\begin{aligned}
& \underline{y}(t ; \alpha)=\alpha(1-\sinh t)+\sinh t-\cos t, \\
& \bar{y}(t ; \alpha)=(2-\alpha)(1-\sinh t)+\sinh t-\cos t,
\end{aligned}
$$

we see that the fuzzy function $y(t)$ has valid level sets for $t \in[0, \ln (1+\sqrt{2})]$ and define a $(2,1)$-solution for the problem on $(0, \ln (1+\sqrt{2}))$.

Finally, to find $(2,2)$-solution, we find

$$
\underline{y}(t ; \alpha)=\alpha(1-\sin t)+\sin t-\cos t, \quad \bar{y}(t ; \alpha)=(2-\alpha)(1-\sin t)+\sin t-\cos t,
$$

that $y(t)$ has valid level sets for $t \geq 0$ and $y$ is $(2,2)$-differentiable on $(0, \pi / 2)$.

We then have a linear fuzzy differential equation with initial condition and two solutions.

\section{Higher-Order Fuzzy Differential Equations}

Selecting different types of derivatives, we get several solutions to fuzzy initial value problem for second-order fuzzy differential equations. Theorem 4.2 has a crucial role in our strategy. To extend the results to $N$ th-order fuzzy differential equation, we can follow the proof of Theorem 4.2 to get the same results for derivatives of higher order. Therefore, we can extend the presented argument for second-order fuzzy differential equation to $N$ th-order. Under generalized derivatives, we would expect at most $2^{N}$ solutions for an $N$ th-order fuzzy differential equation by choosing the different types of derivatives.

\section{Acknowledgments}

We thank Professor J. J. Nieto for his valuable remarks which improved the paper. This research is supported by a grant from University of Tabriz.

\section{References}

[1] A. Kandel and W. J. Byatt, "Fuzzy differential equations," in Proceedings of the International Conference on Cybernetics and Society, pp. 1213-1216, Tokyo, Japan, 1978.

[2] A. Kandel and W. J. Byatt, "Fuzzy processes," Fuzzy Sets and Systems, vol. 4, no. 2, pp. 117-152, 1980.

[3] J. J. Buckley and T. Feuring, "Fuzzy differential equations," Fuzzy Sets and Systems, vol. 110, no. 1, pp. 43-54, 2000.

[4] M. L. Puri and D. A. Ralescu, "Differentials of fuzzy functions," Journal of Mathematical Analysis and Applications, vol. 91, no. 2, pp. 552-558, 1983.

[5] O. Kaleva, "Fuzzy differential equations," Fuzzy Sets and Systems, vol. 24, no. 3, pp. 301-317, 1987.

[6] P. Diamond and P. Kloeden, Metric Spaces of Fuzzy Sets, World Scientific, Singapore, 1994.

[7] E. Hüllermeier, "An approach to modelling and simulation of uncertain dynamical systems," International Journal of Uncertainty, Fuzziness and Knowledge-Based Systems, vol. 5, no. 2, pp. 117-137, 1997.

[8] B. Bede and S. G. Gal, "Almost periodic fuzzy-number-valued functions," Fuzzy Sets and Systems, vol. 147, no. 3, pp. 385-403, 2004. 
[9] B. Bede and S. G. Gal, "Generalizations of the differentiability of fuzzy-number-valued functions with applications to fuzzy differential equations," Fuzzy Sets and Systems, vol. 151, no. 3, pp. 581-599, 2005.

[10] B. Bede, I. J. Rudas, and A. L. Bencsik, "First order linear fuzzy differential equations under generalized differentiability," Information Sciences, vol. 177, no. 7, pp. 1648-1662, 2007.

[11] Y. Chalco-Cano and H. Román-Flores, "On new solutions of fuzzy differential equations," Chaos, Solitons \& Fractals, vol. 38, no. 1, pp. 112-119, 2008.

[12] D. N. Georgiou, J. J. Nieto, and R. Rodríguez-López, "Initial value problems for higher-order fuzzy differential equations," Nonlinear Analysis: Theory, Methods E Applications, vol. 63, no. 4, pp. 587-600, 2005.

[13] J. J. Buckley and T. Feuring, "Fuzzy initial value problem for Nth-order linear differential equations," Fuzzy Sets and Systems, vol. 121, no. 2, pp. 247-255, 2001.

[14] S. S. L. Chang and L. A. Zadeh, "On fuzzy mapping and control," IEEE Transactions on Systems Man Cybernetics, vol. 2, pp. 30-34, 1972.

[15] D. Dubois and H. Prade, "Towards fuzzy differential calculus—part III: differentiation," Fuzzy Sets and Systems, vol. 8, no. 3, pp. 225-233, 1982.

[16] R. Goetschel Jr. and W. Voxman, “Elementary fuzzy calculus,” Fuzzy Sets and Systems, vol. 18, no. 1, pp. 31-43, 1986.

[17] V. Lakshmikantham and J. J. Nieto, "Differential equations in metric spaces: an introduction and an application to fuzzy differential equations," Dynamics of Continuous, Discrete E Impulsive Systems. Series A, vol. 10, no. 6, pp. 991-1000, 2003. 